\title{
POLYFLUOROALKYL-2-HYDROXYIMINO-1,3-DIKETONES AS NEW SELECTIVE CARBOXYLESTERASE INHIBITORS
}

\author{
N.P. Boltneva', S.V. Lushchekina ${ }^{1,2}$, O.G. Serebryakova', E.V. Rudakova', \\ T.Yu. Astakhova' ${ }^{2}$, N.A. Agafonova3 ${ }^{3}$, E.V. Shchegolkov³, N.S. Boltacheva ${ }^{3}$, \\ V.I. Filyakova3 , E.V. Radchenko4, V.A. Palyulin ${ }^{4}$, G.F. Makhaeva', \\ Ya.V. Burgart ${ }^{3}$, V.I. Saloutin ${ }^{3}$, R.J. Richardson ${ }^{5}$ \\ ${ }^{1}$ Institute of Physiologically Active Compounds RAS, \\ 142432, Russia, Chernogolovka, Severny proyezd, 1. \\ 2Emanuel Institute of Biochemical Physics RAS, 119334, Russia, Moscow, Kosygina Str., 4. \\ ${ }^{3}$ Postovsky Institute of Organic Synthesis UB RAS, \\ 620990, Russia, Ekaterinburg, S. Kovalevskoy/Akademicheskaya Str., 22/20. \\ ${ }^{4}$ Department of Chemistry, Lomonosov Moscow State University, \\ 119991, Russia, Moscow, Leninskie gory, 1/3. \\ ${ }^{5}$ Departments of Environmental Health Sciences and Neurology, \\ University of Michigan, 48109 USA Michigan, Ann Arbor.
}

DOI: 10.19163/MedChemRussia2021-2021-256

E-mail: boltneva@ipac.ac.ru

Carboxylesterases (CES, EC 3.1.1.1) are important enzymes of hydrolytic metabolism for major therapeutic agents and other xenobiotics containing an ester group, as well as endogenous esters and thioesters, including lipids. We have shown that the polyfluoroalkyl-2-imino-1,3-dione scaffold is promising for the design of specific CES inhibitors. In this work, a number of polyfluoroalkyl-2-hydroxyimino-1,3diketones (DKs) of the general formula was obtained:

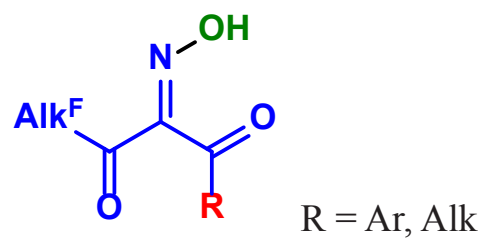

Analysis of the esterase profile demonstrated that DKs effectively and selectively inhibit CES with a very weak inhibition of structurally related cholinesterases. Quantum-mechanical calculations of the DK structure agree with the results of X-ray diffraction analysis. The ${ }^{19} \mathrm{~F}$ NMR and QM calculations showed the ease of hydration of CF3-substituted DKs in polar media, which explains the close anti-CES activity of the ketone and hydrated forms. The mechanism of CES inhibition by diketones and their hydrates was consistent with the results of enzymatic kinetics, molecular docking and QM/MM calculations. All compounds possess high antiradical activity and have favorable ADMET profiles. They are of interest as potential candidates for CES inhibition in biomedical applications.

This work was supported by RFBR 20-03-00312 and Russian State assignment 00902019-0005 to IPAC RAS (biological assay). 